\title{
Anisotropic spin transport in two-terminal mesoscopic rings: the Rashba and Dresselhaus spin-orbit interactions
}

\author{
Miao Wang and Kai Chang* \\ SKLSM, Institute of Semiconductors, Chinese Academy of Sciences, \\ P. O. Box 912, Beijing 100083, China
}

\begin{abstract}
We investigate theoretically the spin transport in two-terminal mesoscopic rings in the presence of both the Rashba spin-orbit interaction (RSOI) and the Dresselhaus spin-orbit interaction (DSOI). We find that the interplay between the RSOI and DSOI breaks the original cylindric symmetry of mesoscopic ring and consequently leads to the anisotropic spin transport, i.e., the conductance is sensitive to the positions of the incoming and outgoing leads. The anisotropic spin transport can survive even in the presence of disorder caused by impurity elastic scattering in a realistic system.
\end{abstract}

PACS numbers: 73.23.-b

*Electronic address: kchang@red.semi.ac.cn 


\section{INTRODUCTION}

In recent years, the spin-orbit interaction (SOI) in low-dimensional semiconductor structures has attracted considerable attention because of its potential application in all-electrical controlled spintronic devices. [1, 2] There are two types of SOI in conventional semiconductors. One is the Rashba spin-orbit interaction(RSOI) induced by structure inversion asymmetry, [3, 4] and the other is the Dresselhaus spin-orbit interaction(DSOI) induced by bulk inversion asymmetry [5]. The strength of the RSOI can be tuned by external gate voltages or asymmetric doping. In thin quantum wells, the strength of the DSOI is comparable to that of the RSOI. [6] The interplay between the RSOI and DSOI leads to a significant change in the transport property. There are a few works on the effects of the competition between these two types of SOI on the transport properties of 2DEG, [7, 8, 9] especially in mesoscopic rings [10]. The circular photogalvanic effect can be used to separate the contribution of the RSOI and DSOI, and the relative strengths of the RSOI and DSOI can be extracted from the photocurrent. [7] The RSOI and DSOI can interfere in such a way that the spin dependent features disappear even though the individual SOI is still strong, e.g., vanishing spin splitting in the presence of the equal-strength RSOI and DSOI. [7] This cancellation results in extremely long spin relaxation time in specific crystallographic directions, and the disappearance of the beating pattern in SdH oscillation. [9]

Recently, advanced growth techniques have made it possible to fabricate high quality semiconductor rings, [11] which have attracted considerable attention due to the intriguing quantum interference phenomenon arising from their unique topological geometry. The Aharonov-Bohm (AB) and the Aharonov-Casher (AC) effects are typical examples of quantum mechanical phase interference, which have been demonstrated experimentally [12, 13] and theoretically [14] on semiconductor rings. The quantum transport properties through semiconductor ring structures with the RSOI alone have attracted considerable interest. [15, 16, 17, 18, 19, 20] SOIs in semiconductors behave like an in-plane momentumdependent magnetic field and lead to a lifting of spin degeneracy of energy bands. This

effective magnetic field induces a wave phase difference between the upper arm and lower arm, resulting in the oscillation of the conductance. [1, 20, 21] Therefore, the conductance oscillates with increasing the strength of the RSOI.[15, 16] The ring subjected to the DSOI alone shows the exact same oscillation, since the Hamiltonian of the RSOI alone is mathemat- 
ically equivalent to that of the DSOI alone by a unitary transformation. [22] The interplay between the RSOI and DSOI results in a periodic potential in an isolated ring, producing the gap in the energy spectrum, suppressing the persistent currents, [22] and breaking the cylindrical symmetry of mesoscopic rings. This interesting feature leads to the anisotropic spin transport and could be detected using the transport property in an open two-terminal mesoscopic ring. This anisotropic spin transport is a new result, is dominant difference between our work and the previous studies, [15, 16, 17, 18, 19] and should be important for the potential application of spintronic devices.

In this paper, we investigate theoretically the spin transport in two-terminal mesoscopic rings in the presence of both the RSOI and DSOI. We find that the interplay between the RSOI and DSOI leads to a significant change in the transmission, the localization of electrons, and the spin polarization of the current. This interplay weakens and smoothens the oscillation of the conductance, and breaks the original cylindrical symmetry, leading to the anisotropic spin transport. The paper is organized as follows, in Sec. II, we present the theoretical model and formulation. The numerical results and discussions are given in Sec. III. Finally, the conclusion is given in Sec. IV

\section{THEORETICAL MODEL}

A semiconductor mesoscopic ring (see Fig. 1) in the presence of the RSOI and DSOI can be described by the single-particle effective mass Hamiltonian

$$
\begin{aligned}
\hat{H} & =\frac{-\hbar^{2} k^{2}}{2 m^{*}}+\alpha\left(\sigma_{x} k_{y}-\sigma_{y} k_{x}\right) \\
& +\beta\left(\sigma_{x} k_{x}-\sigma_{y} k_{y}\right)+V(r),
\end{aligned}
$$

where the $x$ axis is along the [100] direction, $k=-i \nabla$ is the electron wave vector, $m^{*}$ is the electron effective mass, $\sigma_{i}(i=x, y, z)$ are the Pauli matrices, $\alpha$ is the strength of the RSOI, and $\beta$ is the strength of the DSOI. $V(r)$ is the radial confining potential, which is neglected hereafter since we consider that electrons only occupy the lowest subband in a ring with narrow width. The one-dimensional Hamiltonian of a ring in a dimensionless form in lattice 


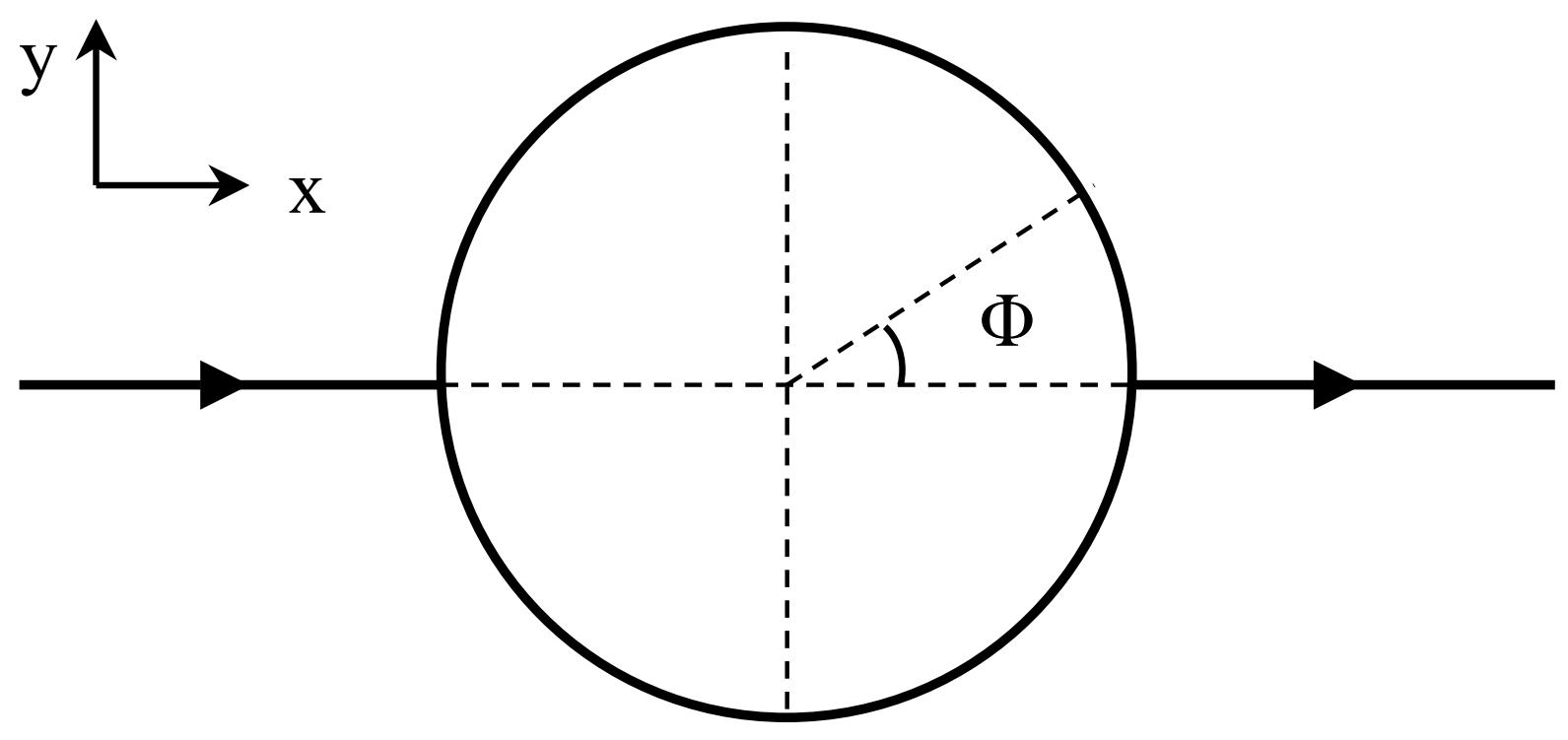

FIG. 1: Schematic diagram of a 1D semiconductor mesoscopic ring with two leads. Electrons are injected from the left lead, pass through the ring, and exit from the right lead. SOI only exists in the ring.

representation is [23]

$$
\begin{aligned}
\hat{H}_{\text {ring }} & =\sum_{n=1}^{N} \sum_{\sigma=\uparrow, \downarrow} \varepsilon_{n} \hat{c}_{n, \sigma}^{\dagger} \hat{c}_{n, \sigma} \\
& -\sum_{n=1}^{N} \sum_{\sigma, \sigma^{\prime}=\uparrow, \downarrow}\left[t_{\phi}^{n, n+1 ; \sigma, \sigma^{\prime}} \hat{c}_{n ; \sigma}^{\dagger} \hat{c}_{n+1 ; \sigma^{\prime}}+\text { h.c. }\right]
\end{aligned}
$$

where the hopping energies are given in the $2 \times 2$ matrix form as:

$$
\begin{aligned}
t_{\phi}^{n, n+1} & =t \hat{I}_{s}-i \frac{\alpha}{2 a}\left(\cos \phi_{n, n+1} \sigma_{x}+\sin \phi_{n, n+1} \sigma_{y}\right) \\
& -i \frac{\beta}{2 a}\left(\cos \phi_{n, n+1} \sigma_{y}-\sin \phi_{n, n+1} \sigma_{x}\right)
\end{aligned}
$$

where $\phi$ is the angular coordinate and $\varepsilon_{n}$ is the on-site potential energy. The operator $\hat{c}_{n, \sigma}\left(\hat{c}_{n, \sigma}^{\dagger}\right)$ annihilates (creates) a spin $\sigma$ electron at the site $n$ of the ring. $\phi_{n, n+1}$ is the angle between the $n$-th site and the $n+1$-th site. $t=\hbar^{2} / 2 m^{*} a^{2}$, with $a$ being the lattice spacing constant, is the nearest-neighbor hopping term in the lead.

The spin-resolved conductance of a two-terminal device can be obtained by using the 
Landauer-Büttiker's formula [24]:

$$
\mathbf{G}=\left(\begin{array}{ll}
G_{\uparrow \uparrow} & G_{\uparrow \downarrow} \\
G_{\downarrow \uparrow} & G_{\downarrow \downarrow}
\end{array}\right)=\frac{e^{2}}{h} \sum_{p, p^{\prime}=1}^{M}\left(\begin{array}{ll}
\left|\mathbf{t}_{p p^{\prime}, \uparrow \uparrow}\right|^{2} & \left|\mathbf{t}_{p p^{\prime}, \uparrow \downarrow}\right|^{2} \\
\left|\mathbf{t}_{p p^{\prime}, \uparrow \uparrow}\right|^{2} & \left|\mathbf{t}_{p p^{\prime}, \downarrow \downarrow}\right|^{2}
\end{array}\right),
$$

where $M$ is the number of conducting channels, the transmission matrix elements $\mathbf{t}=$ $2 \sqrt{-\operatorname{Im} \sum_{L}^{r} \otimes I_{s}} \cdot G_{1 N}^{r} \cdot \sqrt{-\operatorname{Im} \sum_{R}^{r} \otimes I_{s}}$ and $\left|\mathbf{t}_{n n^{\prime}, \sigma \sigma^{\prime}}\right|^{2}$ represents the probability for a spin- $\sigma$ electron incoming from the left lead in the orbital state $|n\rangle$ to appear as a spin- $\sigma^{\prime}$ electron in the orbital channel $\left|n^{\prime}\right\rangle$ in the right lead.

We can calculate the conductance from lead $p$ to lead $q$ by using the Fisher-Lee relation [25]. The detailed formula can be found in the Ref. 26:

$$
\begin{gathered}
\mathbf{G}^{R}=\left[E I-H_{c}-\Sigma^{R}\right]^{-1}, \\
\overline{\mathbf{T}}_{p q}=\operatorname{Tr}\left[\Gamma_{p} G^{R} \Gamma_{q} G^{A}\right],
\end{gathered}
$$

where $H_{c}$ is the Hamiltonian of the $1 \mathrm{D}$ isolated ring. $\Gamma_{p}(i, j)=\sum_{m} \chi_{m}\left(p_{i}\right) \frac{\hbar v_{m}}{a} \chi_{m}\left(p_{j}\right)$ describes the coupling of the ring conductor to the leads. We assume the RSOI and DSOI only exist in the ring, and are absent in the leads. The self-energy $\Sigma^{R}=$ $\sum_{p=1,2} \Sigma_{p}^{R}$, where $\Sigma_{p}^{R}(i, j)=t^{2} g_{p}^{R}\left(p_{i}, p_{j}\right)$, describes the effect of the external leads on the ring. The Green's function between two points along the leads is given by $g_{p}^{R}\left(p_{i}, p_{j}\right)=$ $-\frac{1}{t} \sum_{m} \chi_{m}\left(p_{i}\right) \exp \left[i k_{m} a\right] \chi_{m}\left(p_{j}\right)$. The function $\chi_{m}\left(p_{i}\right)$ describes the $m$-th mode in lead $i$. In this paper, we take $a$ as the length unit and $E_{0}=\hbar^{2} / 2 m^{*} a^{2}$ as the energy unit.

The local density of electron states is [26]:

$$
\rho(r, E)=\frac{1}{2 \pi} A(r, r ; E)=-\frac{1}{\pi} \operatorname{Im}\left[G^{R}(r, r ; E)\right],
$$

where $A \equiv i\left[G^{R}-G^{A}\right]$ is the spectral function, which can also be written:

$$
\begin{aligned}
\rho(r, E) & \sim \sum_{n} \frac{1}{2 \pi} \frac{\gamma_{n} \psi_{n}(r) \phi_{n}^{*}(r)}{\left(E-\varepsilon_{n 0}+\Delta_{n}\right)^{2}+\left(\gamma_{n} / 2\right)^{2}} \\
& \rightarrow \sum_{n} \delta\left(E-\varepsilon_{n 0}\right)\left|\psi_{n}(r)\right|^{2} \quad \text { as } \quad \gamma_{n} \rightarrow 0
\end{aligned}
$$

where $\hbar / 2 \gamma_{n}$ represents the lifetime of an electron remaining in state $n$ before it escapes into the leads, $\varepsilon_{n 0}$ is the eigenenergy of the isolated conductor, and $\psi(\phi)$ is the eigenstates of the effective Hamiltonian $\left[H_{c}+\Sigma^{R}\right]\left(\left[H_{c}+\Sigma^{A}\right]\right)[26]$. 


\section{RESULTS AND DISCUSSIONS}

\section{A. 1D ring with both RSOI and DSOI}

Many previous works investigating the spin transport through a 1D ring account only for the RSOI. [15] The RSOI behaves like an effective in-plane momentum-dependent magnetic field. This effective magnetic field induces a phase difference between the electrons traveling clockwise and counterclockwise along the ring's upper and lower arms. Therefore, the conductance of a $1 \mathrm{D}$ ring in the presence of the RSOI oscillates quasi-periodically with changing the strength of the RSOI and the Fermi energy $E_{F}$.

We study the transport through a mesoscopic ring in the presence of both the RSOI and DSOI. First, we consider the ballistic transport through the mesoscopic ring in the presence of the RSOI(DSOI) alone. In Fig. 2, we plot the conductance through a 1D ring as a function of the strength of the RSOI $Q_{r}$. This figure shows that the conductances are exactly same when the right lead is located at symmetric positions, e.g., $\phi= \pm \frac{1}{4} \pi, \pm \frac{1}{2} \pi$, and $\pm \frac{3}{4} \pi$. The RSOI or DSOI alone in the ring does not break the cylindrical symmetry and the transport is still isotropic when the outgoing leads are located at symmetric positions with respect to the $x$-axis (see the dashed lines in the insets of Fig. 2). The quantum interference between the alternation paths, the spin-up or spin-down clockwise and anticlockwise, is responsible for the oscillation of the conductance.

When the 1D mesoscopic ring is subjected to both the RSOI and DSOI, as shown in Fig. 3, the conductances become asymmetric when the outgoing lead is located at symmetric positions, e.g., $\phi= \pm \frac{1}{4} \pi, \pm \frac{1}{2} \pi$, and $\pm \frac{3}{4} \pi$. The anisotropy of the conductance is induced by the interplay of the RSOI and DSOI, which leads to a periodic potential $\frac{\alpha \beta}{2} \sin 2 \phi$. [22] The height of the periodic potential is determined by the product of the strengths of the RSOI and DSOI, and the periodicity of the potential is fixed at $\pi$. The potential exhibits barriers at $\phi=\frac{1}{4} \pi,-\frac{3}{4} \pi$, and the valleys at $\phi=-\frac{1}{4} \pi, \frac{3}{4} \pi$. Thus, the conductance displays asymmetric features for the symmetric positions of the outgoing leads.

If the incoming lead locates at $\phi=\frac{3}{4} \pi$ (see Fig. 4), we find the transmission becomes symmetric for the outgoing lead locating at the symmetric positions respect to the new incoming lead. In Fig. 4, we plot the conductance of a 1D ring with the incoming lead located at $\phi=\frac{3}{4} \pi$. The conductance becomes symmetric again with respect to the straight 

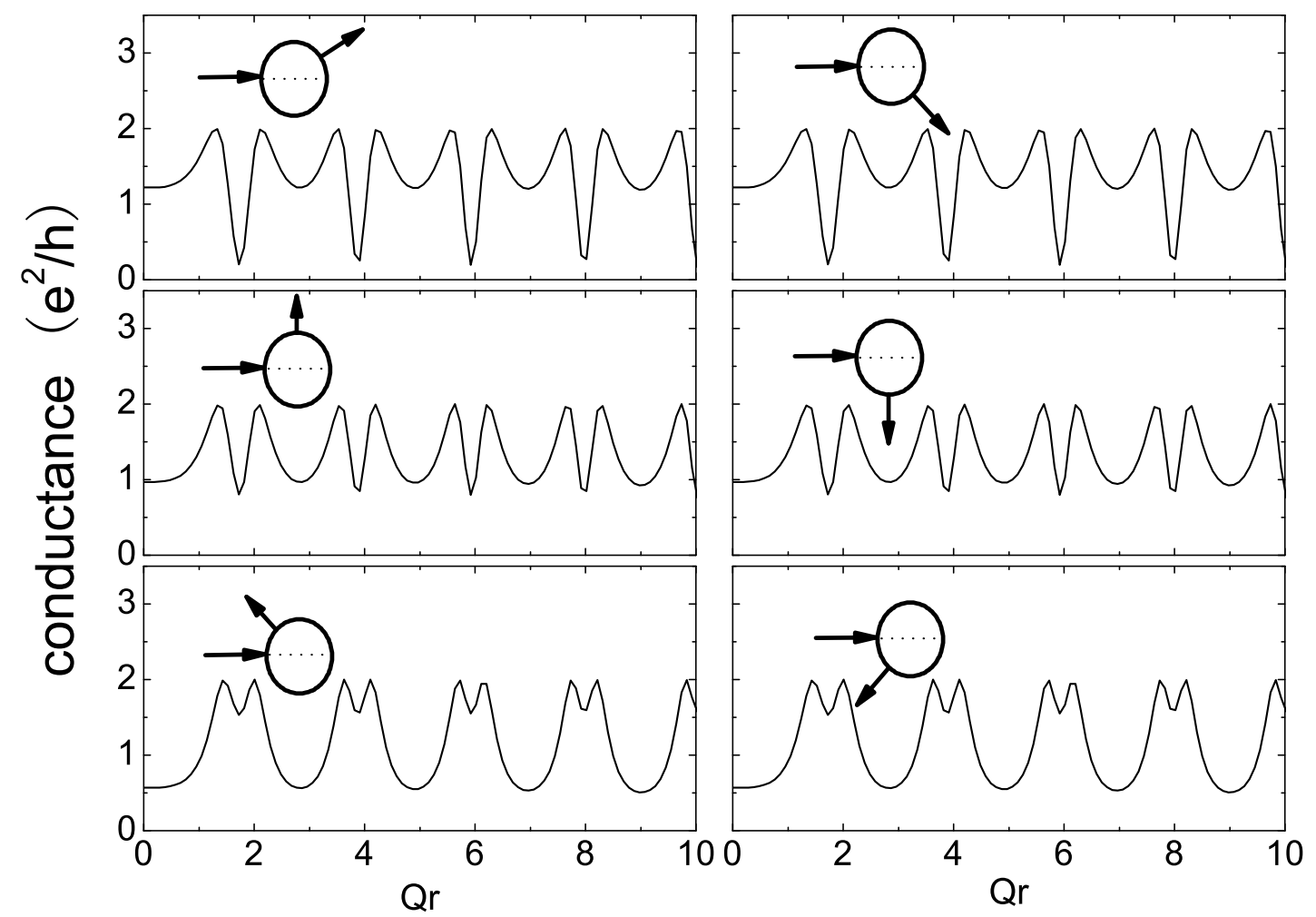

FIG. 2: The conductance through a $1 \mathrm{D}$ ring in the presence of the RSOI or DSOI alone as a function of the strength of the RSOI $Q_{r} \equiv \alpha N / 2 \operatorname{ta\pi }, E_{F}=-0.1$, and the outgoing lead is located at $\pm \frac{1}{2} \pi, \pm \frac{1}{4} \pi, \pm \frac{3}{4} \pi$, respectively (see the insets).

line $\phi=\frac{3}{4} \pi$ and $\phi=-\frac{1}{4} \pi$ (the dashed lines in the insets of Fig. 41). The periodic potential $\frac{\alpha \beta}{2} \sin 2 \phi$ induced by the interplay between the RSOI and DSOI [22] results in the maxima at $\phi=\frac{1}{4} \pi,-\frac{3}{4} \pi$, and the minima at $\phi=\frac{3}{4} \pi,-\frac{1}{4} \pi$.

In order to describe the magnitude of the anisotropy of the conductance induced by the interplay of the RSOI and DSOI, we define the ratio $\eta$ as:

$$
\eta(\phi,-\phi)=\frac{G_{\phi}-G_{-\phi}}{\left(G_{\phi}+G_{-\phi}\right) / 2}
$$

where $G_{ \pm \phi}$ is the conductance when the right lead is located at the positions with an angle $\pm \phi$ with respect to the $x$ axis.

In Fig. 5, we plot $\eta(\pi / 4,-\pi / 4)$ as a function of the strength of the RSOI and DSOI when the left lead is located at the position of $\phi=\pi$. $\eta$ oscillates with the changing strength 

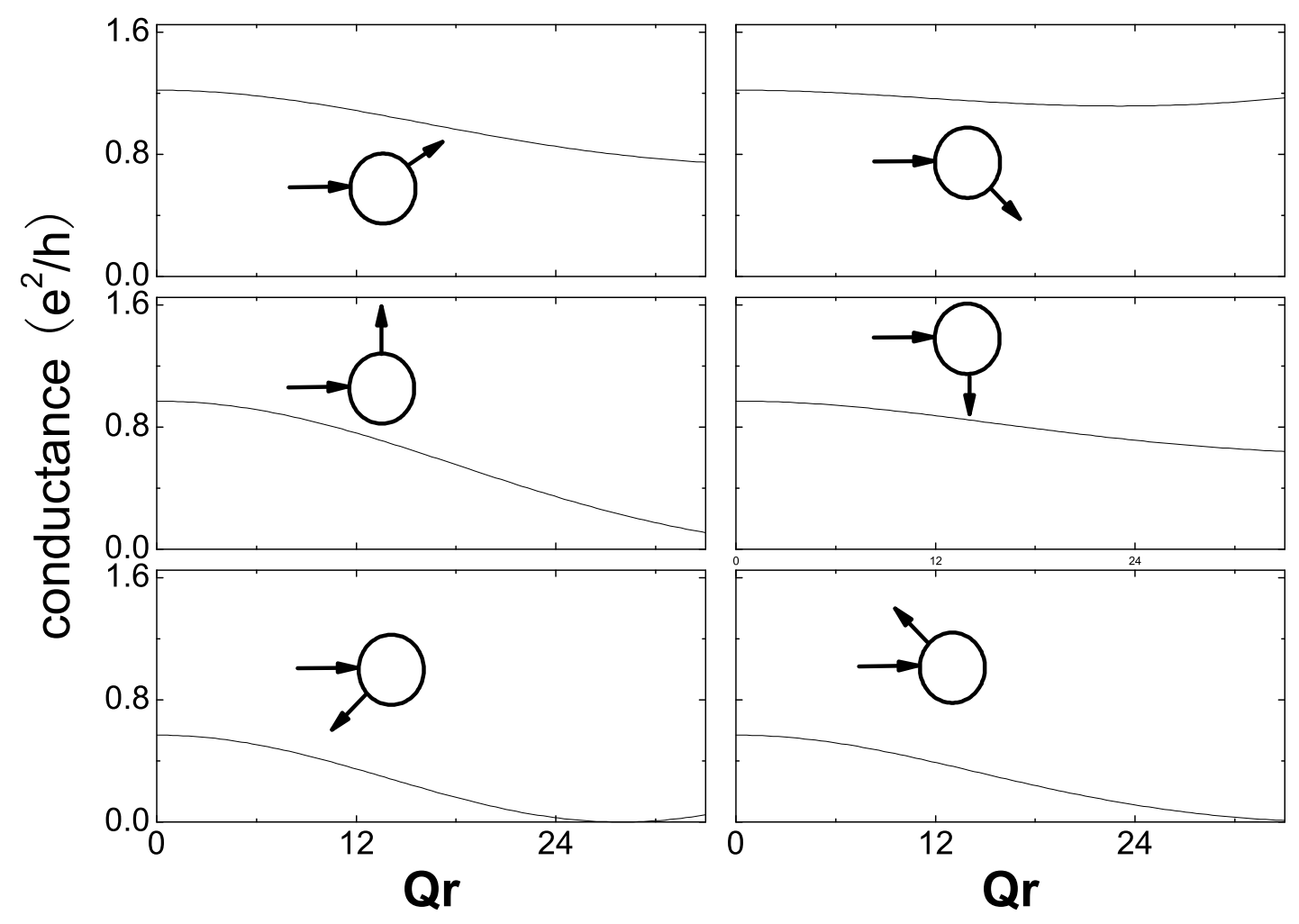

FIG. 3: The conductance through a 1D ring in the presence of the RSOI and DSOI as a function of the strength of the RSOI and DSOI, $Q_{d} \equiv \beta N / 2 t a \pi, Q_{r}=Q_{d}, E_{F}=-0.1$. The outgoing lead is located at $\pm \frac{1}{2} \pi, \pm \frac{1}{4} \pi, \pm \frac{3}{4} \pi$ respectively.

of the RSOI and DSOI. The maximum of the anisotropy of the conductance can approach $20 \%$. This anisotropic transport can be interpreted as follows. The interplay between the RSOI and DSOI leads to an effective periodic potential $\frac{\alpha \beta}{2} \sin 2 \phi$. [22] The potential height is related to the strength of the RSOI and DSOI. $\eta(\phi,-\phi)=0$ when the ring subjected to the DSOI alone because the periodic potential $\frac{\alpha \beta}{2} \sin 2 \phi$ disappears when $\alpha=0$. This effective periodic potential exhibits the maxima at $\phi=\frac{1}{4} \pi$, and $-\frac{3}{4} \pi$, and the minima at $\phi=-\frac{1}{4} \pi$, and $\frac{3}{4} \pi$. Therefore, the interplay between RSOI and DSOI breaks the cylindrical symmetry of the ring (see Fig. 7).

In order to clarify the effect of the invasive role of the lead on the anisotropy of the spin transport, we consider different strengths between the ring and leads (as shown in 

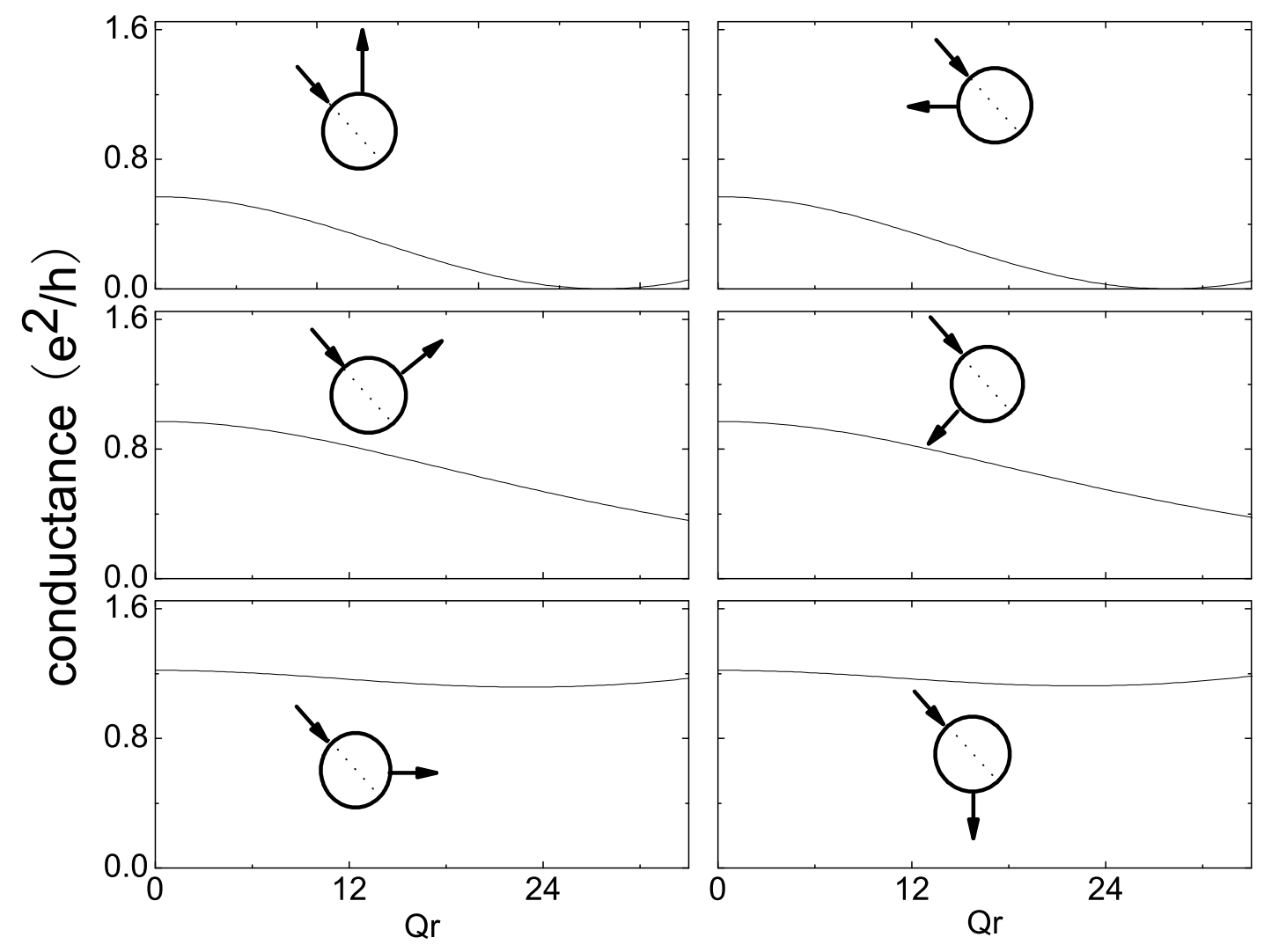

FIG. 4: Same as Fig. 3, but the incoming lead is located at $\phi=\frac{3}{4} \pi$, and the outgoing lead is located at $\phi=0, \pm \frac{1}{2} \pi, \frac{1}{4} \pi,-\frac{3}{4} \pi, \pi$, respectively.

Fig. 6). We find that the conductance decreases with decreasing the coupling strength, but the anisotropy ratios are almost same as before. We believe that the anisotropic spin transport property is caused by the interplay between the Rashba and Dresselhaus spin-orbit interactions.

Fig. 7 describes how the conductance varies with the variation of the strengths of the RSOI and DSOI. The conductance oscillates quasiperiodically as the strengths of the RSOI and DSOI increase, and is symmetric with respect to the straight line $\alpha=\beta$, since the Hamiltonian of the RSOI and that of the DSOI are equivalent and can be transferred by the $S U(2)$ unitary transformation. The contribution from the RSOI and DSOI to the spin splitting of electrons cancel each other, [22] which results in the disappearance of the oscillation along $\alpha=\beta$. This feature provides a possible way to detect the strength of the DSOI 


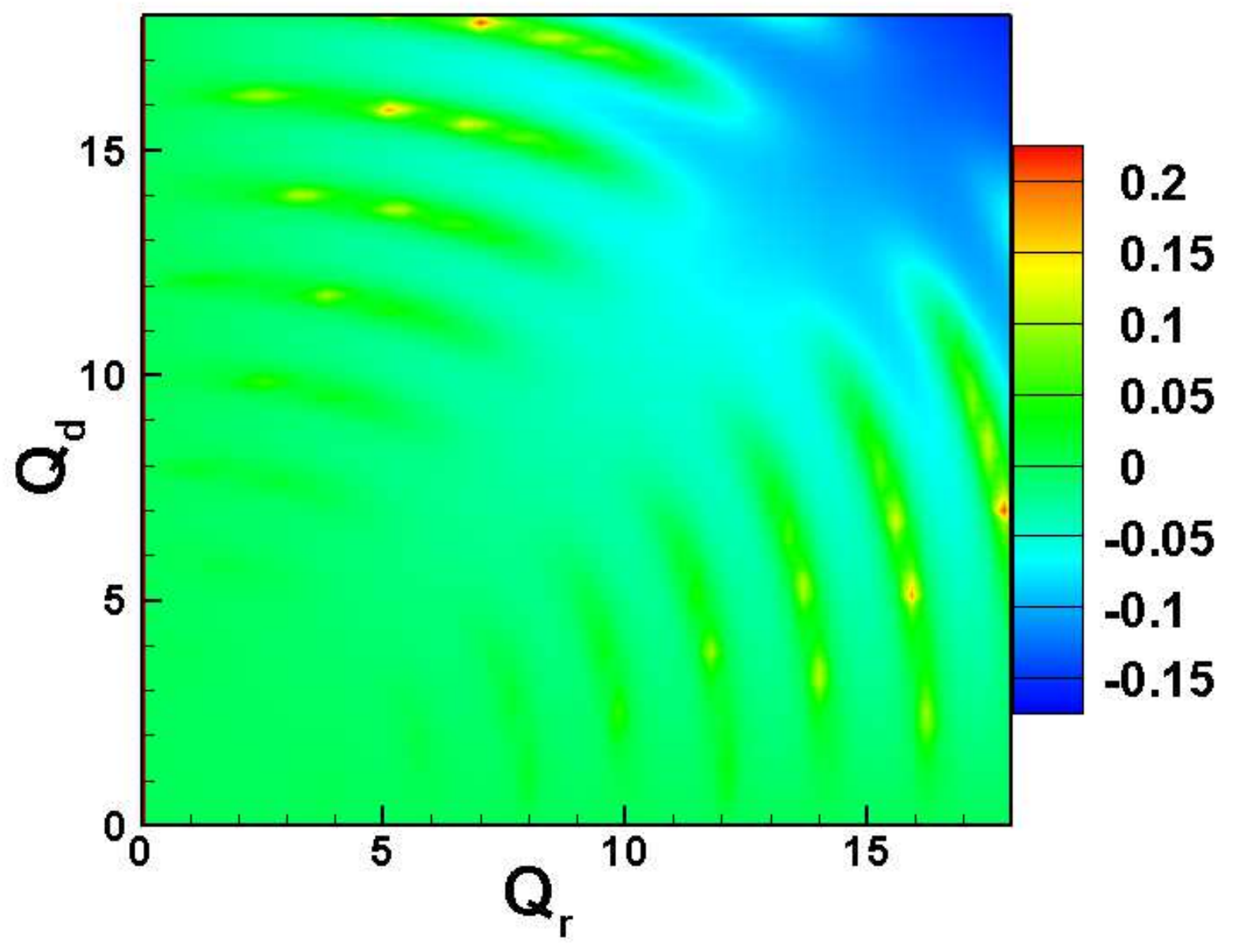

FIG. 5: (Color online) The ratio $\eta$ as a function of the strength of the RSOI $Q_{r}$ and DSOI $Q_{d}$, when $E_{F}=-0.1$. The incoming lead is located at $\phi=\pi$, and the outgoing lead is located at $\phi=\pi / 4,-\pi / 4$, respectively.

since the strength of the RSOI can be tuned by the external electric fields.

Below, we demonstrate that the interplay between the RSOI and DSOI also results in the variation of the local density of electrons in the ring. In Fig. 8, we plot the local density of electrons in the ring from Eq. 7 with and without the SOI. Fig. 8(a) and (b), shows that the local density of electrons shows slow and very rapid oscillations. The fast oscillation comes from the contribution of each site of the lattice, while the slow variation of the envelope corresponds to the bound (quasibound) states in the isolated (open) ring. This feature is analogous to the situation of the effective mass theory, where the electron wave function can be expressed as the product of two parts: the band-edge Bloch function and the slow varying 


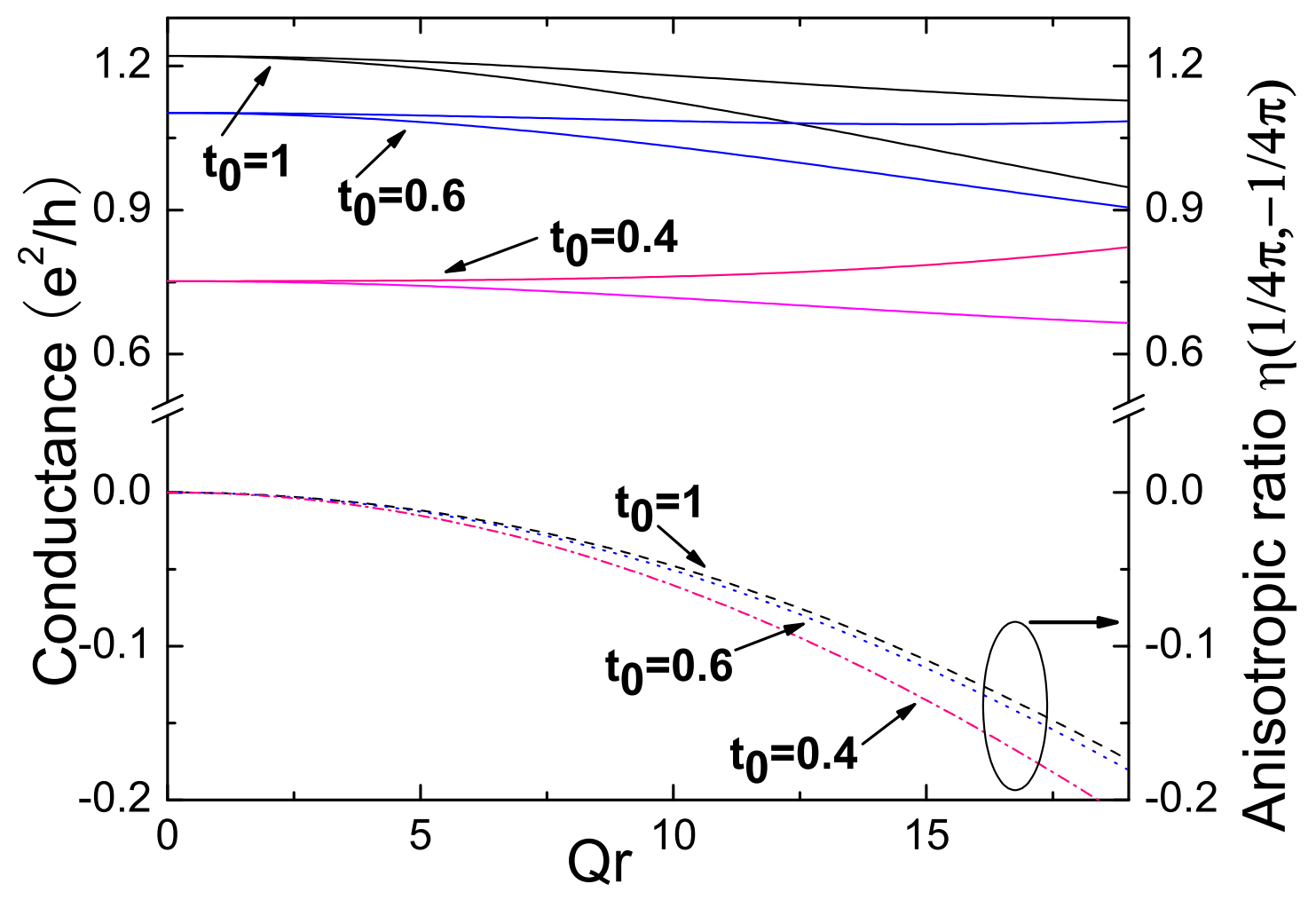

FIG. 6: (Color online) The conductance of $1 \mathrm{D}$ ring as a function of the strength of equal RSOI and DSOI, when $E_{F}=-0.1$ for different coupling strengths $t_{0}=1,0.6,0.4$.

envelope function. The former denotes the contribution from the atomic wave function, and the latter describes the bound (quasibound) state from the external potential, e.g., the quantum well potential. Similar results can be found in Ref. 27.

There is only a slight difference between the local densities of electron states with and without the RSOI, but a significant change in the presence of both the RSOI and DSOI (see Fig. $8(\mathrm{c})$ ). The local density of electrons exhibit maxima at $\phi=-\frac{1}{4} \pi, \frac{3}{4} \pi$. This characteristic is also caused by the periodic potential induced by the interplay between the RSOI and DSOI. The positions of $\phi=\frac{1}{4} \pi,-\frac{3}{4} \pi\left(\phi=-\frac{1}{4} \pi, \frac{3}{4} \pi\right)$ correspond to a potential barrier (well), where the local density of electron states is smaller (larger). The interplay between the RSOI and DSOI induces periodic potential and breaks the original cylindrical symmetry of the ring, consequently changing the local density of electron states. 


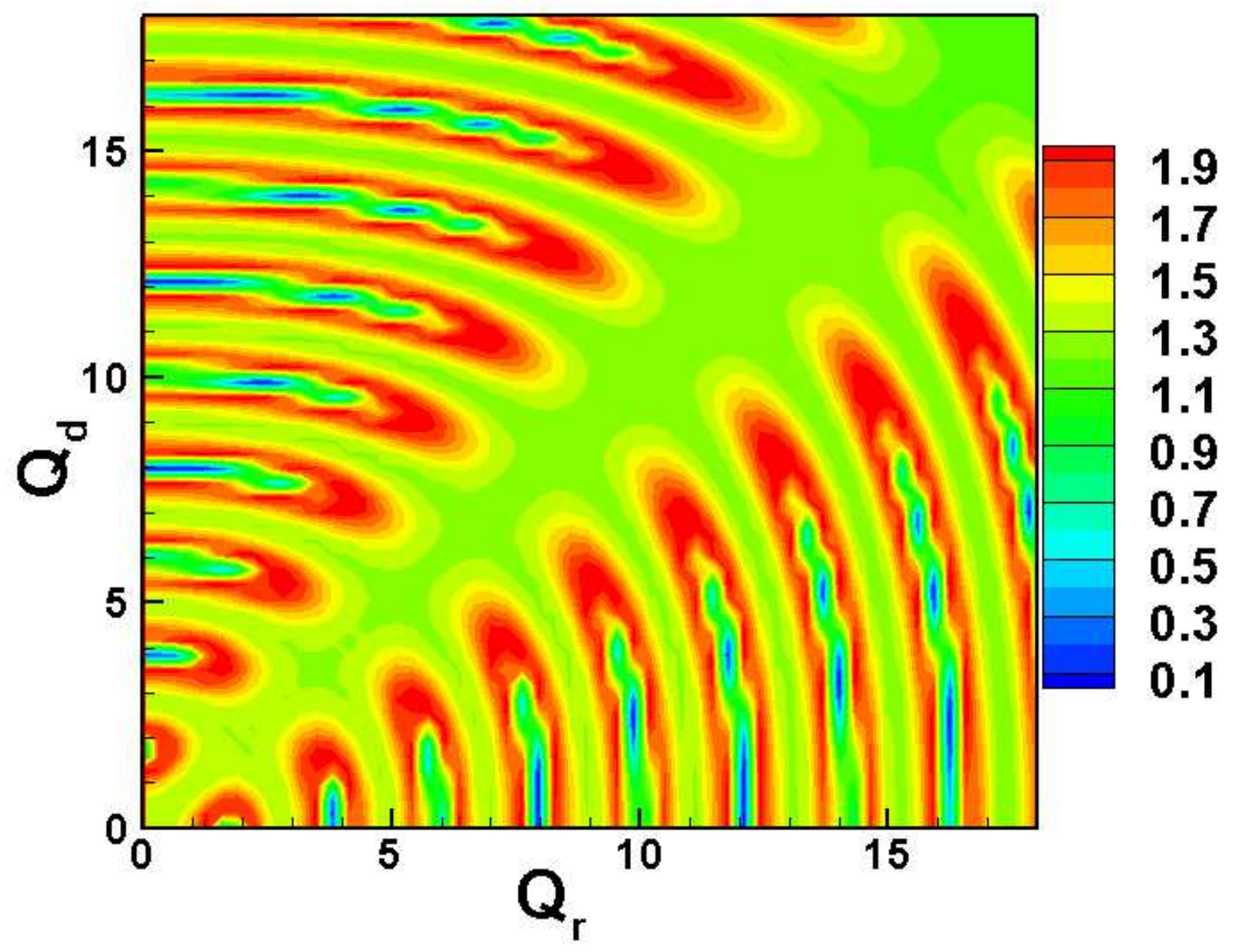

FIG. 7: (Color online) The conductance of a 1D ring as a function of the strength of the RSOI $Q_{r}$ and DSOI $Q_{d}$, when $E_{F}=-0.1$. The incoming lead is located at $\phi=\pi$, while the outgoing lead is located at $\phi=0$.

The above analysis assumes perfectly clean 1D systems, in which there is no elastic or inelastic scattering at $T=0$. In a realistic system, there will be many impurities in the sample. Disorder could be incorporated by the fluctuation of the on-site energies, which distribute randomly within the range width $w\left[\varepsilon_{n} \rightarrow \varepsilon_{n}+w_{n}\right.$ with $\left.-w / 2<w_{n}<w / 2\right]$.

In Fig. 9(a), we plot the conductance as a function of Fermi energy $E_{F}$ without RSOI. The ratio $\eta\left(\frac{1}{4} \pi,-\frac{1}{4} \pi\right)$ is negligible for (weak and strong) different disorders $w=0.1,0.3$ when the system is without the RSOI. Fig. 9(b) plots the conductance of a 1D ring as a function of the strength of RSOI and DSOI, when $Q_{r}=Q_{d}$, for the various random widths $w=0.1,0.3,1$ ( $w=1$ for inset). It can be clearly seen that the disorder-averaged conductance for the 


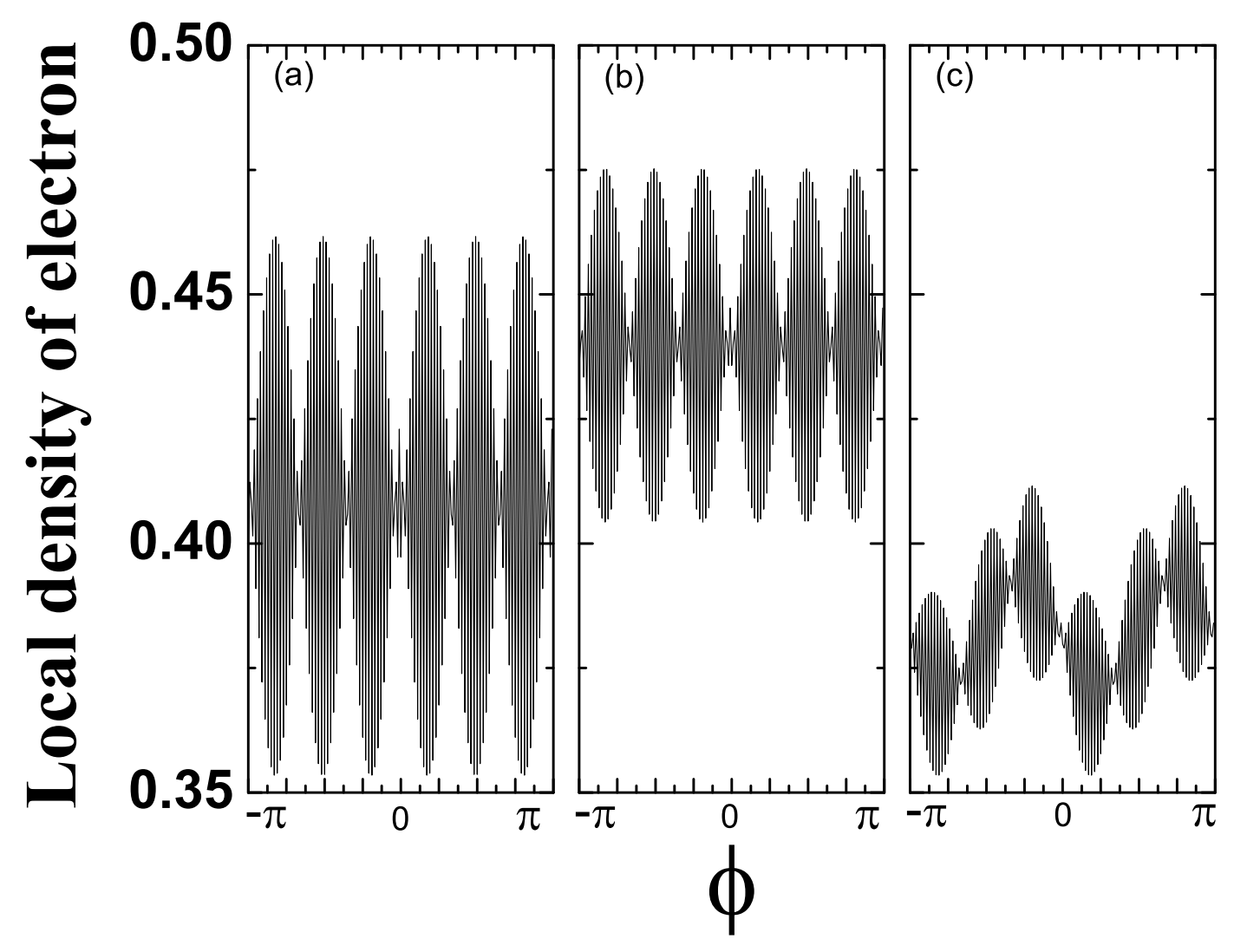

FIG. 8: The local density of electrons along the $\operatorname{ring} \phi$ when $E_{F}=0.1$ (a) without the RSOI and DSOI; (b) with the RSOI alone; (c) with equal RSOI and DSOI ( $Q_{r}=Q_{d}=11.3$ ).

strong disorder case $(w=1)$ shows almost the same anisotropy as that for the weak disorder case $(w=0.1,0.3)$. (see Fig. 9(b))

While the anisotropy of the $1 \mathrm{D}$ ring becomes significant as the strengths of the RSOI and DSOI increase, random disorder increases the scattering of the ring, and decreases conductance compared to that of a clean 1D ring. The anisotropic spin transport can still survive even in the presence of weak and strong disorder. 


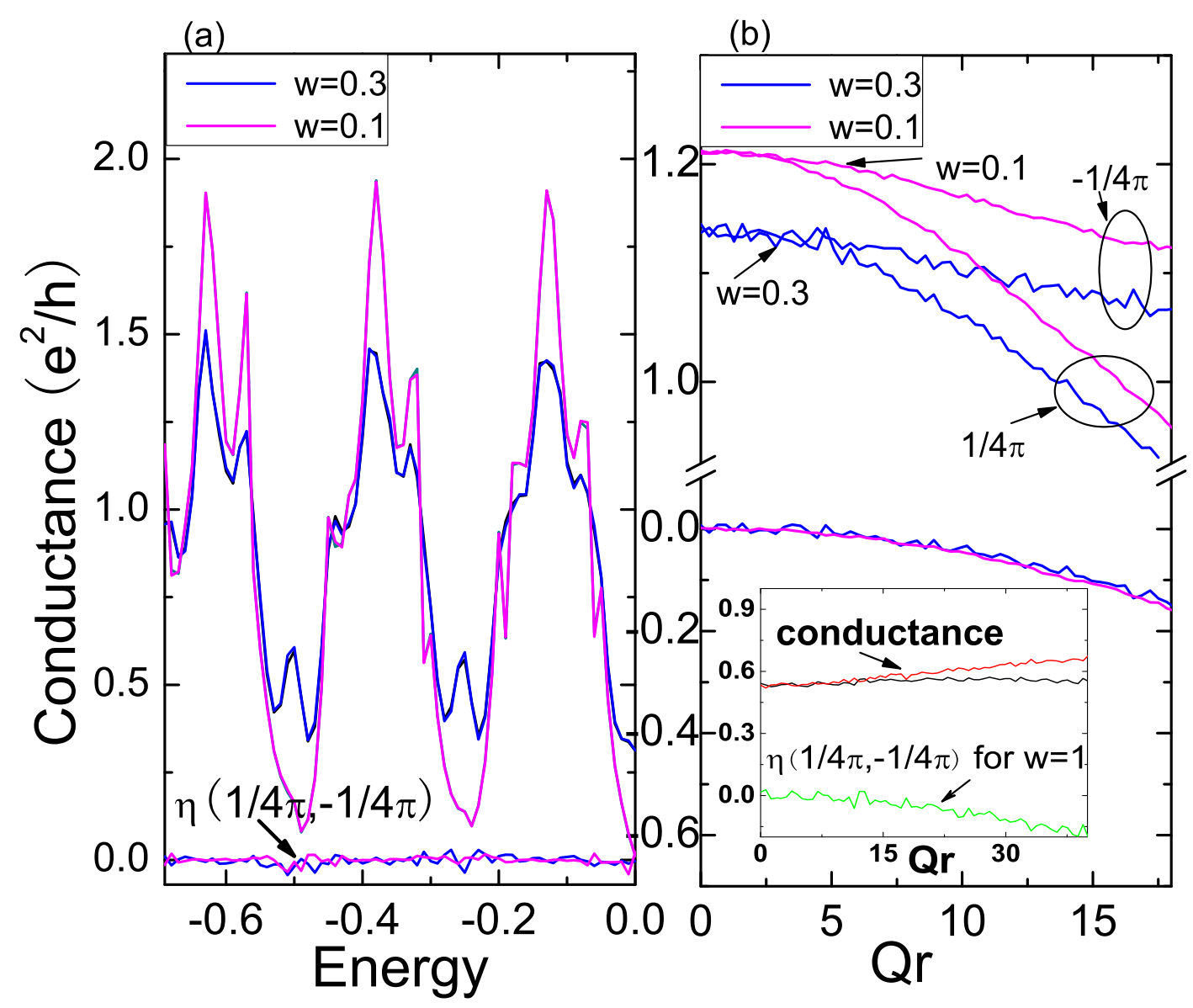

FIG. 9: (Color online)(a) The conductance of a $1 \mathrm{D}$ ring as a function of Fermi energy $E_{F}$ without SOIs for random width $w=0.1,0.3$; (b) The conductance and $\eta$ of a $1 \mathrm{D}$ ring as a function of the strength of the RSOI, for outgoing lead located at $\phi=\frac{1}{4} \pi,-\frac{1}{4} \pi$, and $Q_{r}=Q_{d}, E_{F}=0.1$, $w=0.1,0.3$. The inset shows the conductance and the anisotropic ratio $\eta$ when $w=1$.

\section{B. The spin polarization of current}

The spin polarization vector of current $\boldsymbol{P}=\left(P_{x}, P_{y}, P_{z}\right)$ can be evaluated as follows [23, 24]:

$$
\boldsymbol{P}^{\sigma}=\operatorname{Tr}_{s}\left[\hat{\rho}^{\sigma} \hat{\boldsymbol{\sigma}}\right]
$$


where the density matrix is given by:

$$
\hat{\rho}^{\sigma}=\frac{e^{2} / h}{G^{\uparrow \sigma}+G^{\downarrow \sigma}} \sum_{p, p^{\prime}=1}^{M}\left(\begin{array}{cc}
\left|\mathbf{t}_{p p^{\prime}, \uparrow \sigma}\right|^{2} & \mathbf{t}_{p p^{\prime}, \uparrow \sigma} \mathbf{t}_{p p^{\prime}, \downarrow \sigma}^{*} \\
\mathbf{t}_{p p^{\prime}, \downarrow \sigma} \mathbf{t}_{p p^{\prime}, \uparrow \sigma}^{*} & \left|\mathbf{t}_{p p^{\prime}, \downarrow \sigma}\right|^{2}
\end{array}\right),
$$

where $\operatorname{Tr}_{s}$ denotes the trace in the spin Hilbert space. Then, the spin polarized vector $\boldsymbol{P}$ is [23]:

$$
\begin{aligned}
& P_{x}^{\sigma}=\frac{G^{\uparrow \sigma}-G^{\downarrow \sigma}}{G^{\uparrow \sigma}+G^{\downarrow \sigma}}, \\
& P_{y}^{\sigma}=\frac{2 e^{2} / h}{G^{\uparrow \sigma}+G^{\downarrow \sigma}} \sum_{p, p^{\prime}=1}^{M} \operatorname{Re}\left[\mathbf{t}_{p p^{\prime}, \uparrow \sigma} \mathbf{t}_{p p^{\prime}, \downarrow \sigma}^{*}\right], \\
& P_{z}^{\sigma}=\frac{2 e^{2} / h}{G^{\uparrow \sigma}+G^{\downarrow \sigma}} \sum_{p, p^{\prime}=1}^{M} \operatorname{Im}\left[\mathbf{t}_{p p^{\prime}, \uparrow \sigma} \mathbf{t}_{p p^{\prime}, \downarrow \sigma}^{*}\right],
\end{aligned}
$$

where the $x$-axis is chosen as the spin-quantized axis, $\hat{\sigma}_{x}|\uparrow\rangle=+|\uparrow\rangle$ and $\hat{\sigma}_{x}|\downarrow\rangle=-|\downarrow\rangle$, so that Pauli spin matrix has the following form:

$$
\hat{\sigma}_{x}=\left(\begin{array}{cc}
1 & 0 \\
0 & -1
\end{array}\right), \hat{\sigma}_{y}=\left(\begin{array}{ll}
0 & 1 \\
1 & 0
\end{array}\right), \hat{\sigma}_{z}=\left(\begin{array}{cc}
0 & i \\
-i & 0
\end{array}\right) .
$$

For the spin polarized injection, i.e., $P_{x}=1$, the magnitude of the spin polarization $P$ in the outgoing lead will not change, i.e., $|P|=1$ since there is no other orbit channel to interact with the spin.[28]

Fig. 10 depicts the current spin polarization $P_{i}(i=x, y, z)$ of a $1 \mathrm{D}$ ring as a function of the strength of the RSOI $Q_{r}$ and the positions of the outgoing lead. The RSOI behaves like an effective in-plane momentum-dependent magnetic field, and the fully spin-up polarized current in the incoming lead will be changed to the spin-down current in the outgoing lead at large RSOI. The three components of the outgoing polarization vector also show cylindrical symmetry for the RSOI or DSOI alone, since the RSOI or DSOI alone does not break the cylindrical symmetry of a 1D ring. The spin polarization $P_{x}$ decreases rapidly from $P_{x}=1$ to $P_{x} \approx-1$ as the strength of the RSOI increases when the outgoing lead is located at the position near $\phi=0$, while the spin polarization $P_{y}$ and $P_{z}$ oscillate and decrease to zero. When the outgoing lead locates away from the $x$-axis, i.e., $\phi=0, P_{y}$ and $P_{z}$ oscillate quickly with increasing $Q_{r}$.

In Fig. 11, we show how the spin polarizations $P_{i}(i=x, y, z)$ vary with the strength of the SOIs and the position of the outgoing lead $\phi$ in the presence of equal-strength RSOI 
(a) $\mathrm{Px}$

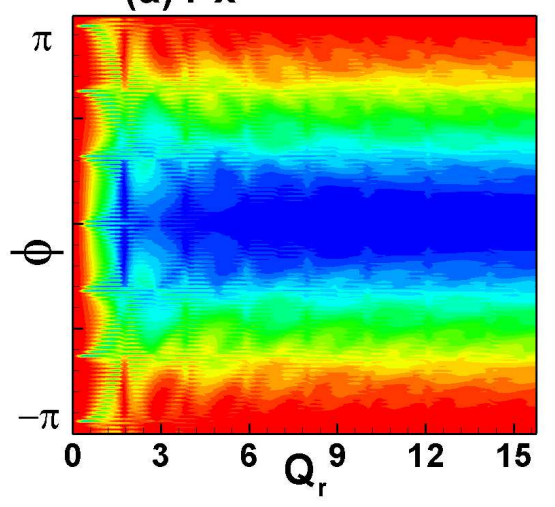

(b) Py

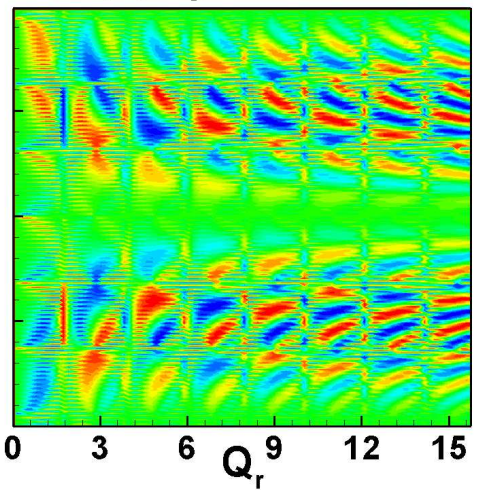

(c) $\mathrm{Pz}$

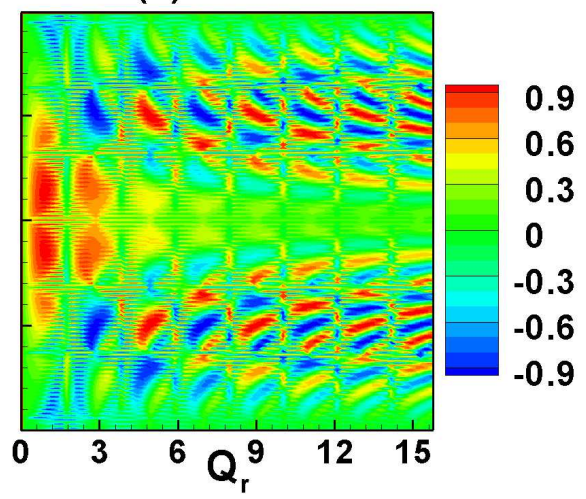

FIG. 10: (Color online) The contour plot of the spin polarization of current as a function of the strength of the RSOI $Q_{r}$ alone and the position of the right lead in the absence of the DSOI, $E_{F}=-0.1, Q_{d}=0$. (a) for $P_{x}$; (b) for $P_{y}$; (c) for $P_{z}$. The spin-quantized axis is the $x$-axis.

and DSOI, i.e., $Q_{r}=Q_{d}$. All three components $P_{x}, P_{y}$, and $P_{z}$ oscillate regularly as the strengths of the RSOI and DSOI increase, and show significant anisotropy of spin polarization with respect to the position of the outgoing lead. This feature can also be understood from the interplay between the effective periodic potential induced by the SOIs and the quantum interference. For a fixed strength of the SOI, the asymmetric characteristic of the polarization $\mathbf{P}$ as a function of the angle $\phi$ arises from the cylinder symmetry breaking induced by the effective potential $\frac{\alpha \beta}{2} \sin 2 \phi$. The quantum interference between the spin -up and -down electrons traveling clockwise and/or counterclockwise along the ring's upper and lower arms leads to the oscillation of the polarization $\mathbf{P}$ as a function of the strengths of the SOIs at a fixed angle $\phi$. Compared to Fig. 10, the spin polarization $P_{x}$ will decrease to 0 instead of -1 as the strengths of the SOIs increase. This is because the DSOI behaves like a twisted in-plane magnetic field, while the effective magnetic field induced by the RSOI always points along the radial of the ring.

\section{CONCLUSION}

We investigate theoretically the spin transport through a two-terminal mesoscopic ring in the presence of both the RSOI and DSOI. We find that the interplay between the RSOI and DSOI leads to the anisotropic transport through a two-terminal cylindrical mesoscopic ring, i.e., breaks the cylindrical symmetry. This interesting feature arises from the peri- 
(a) Px

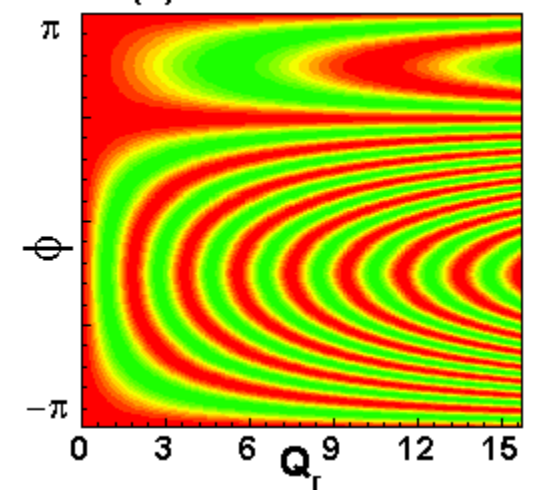

(b) Py

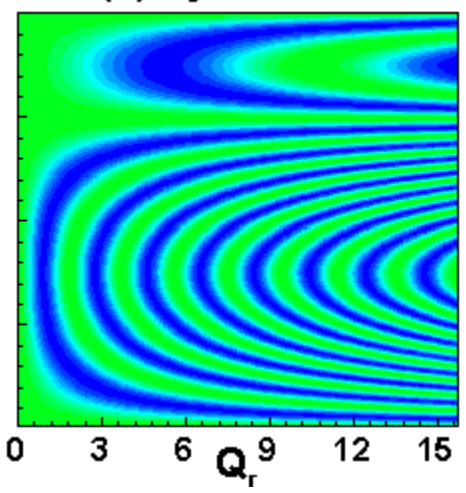

(c) Pz

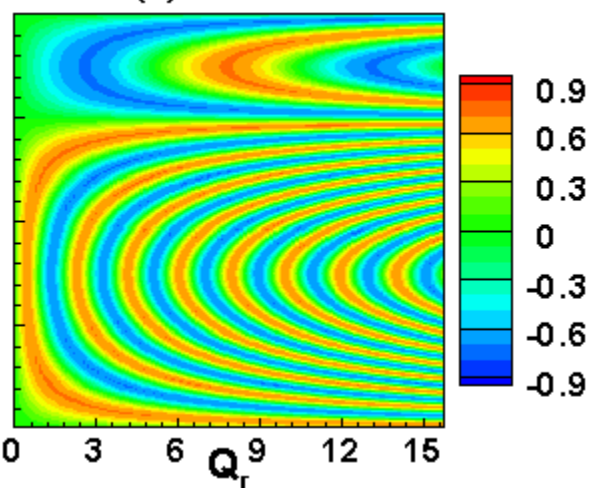

FIG. 11: (Color online) The same as Fig. 10, but includes the DSOI.

odic potential along the ring caused by the interplay between the RSOI and DSOI. This interplay also results in a significant variation in electron density and the spin polarization of current. The anisotropy of the spin transport through the mesoscopic ring induced by the interplay between the RSOI and DSOI can survive even in the presence of the disorder effect. Furthermore, the anisotropy of the spin transport should play an important role in the potential application of all-electrical spintronic devices.

\section{Acknowledgments}

This work is partly supported by NSFC Grant No. 62525405 and the knowledge innovation project of CAS.

[1] S. A. Wolf, D. D. Awschalom, R. A. Buhrman, J. M. Daughton, S. von Molnár, M. L. Roukes, A. Y. Chtchelkanova, and D. M. Treger, Science 294, 1488 (2001).

[2] E. Tsitsishvili, G. S. Lozano, and A. O. Gogolin, Phys. Rev. B 70, 115316 (2004).

[3] E. I. Rashba, Sov. Phys. Solid State 2, 1109 (1960).

[4] Y. A. Bychkov and E. I. Rashba, J. Phys. C 17, 6039 (1984).

[5] G. Dresselhaus, Phys. Rev. 100, 580 (1955).

[6] G. Lommer, F. Malcher, and U. Rössler, Phys. Rev. Lett. 60, 728 (1988).

[7] S. D. Ganichev, V. V. Bel'kov, L. E. Golub, E. L. Ivchenko, P. Schneider, S. Giglberger, J. 
Eroms, J. De Boeck, G. Borghs, W. Wegscheider, D. Weiss, and W. Prettl, Phys. Rev. Lett. 92, 256601 (2004).

[8] M. C. Chang, Phys. Rev. B 71, 085315 (2005).

[9] W. Yang and K. Chang, Phys. Rev. B 73, 045303 (2006).

[10] X. F. Wang and P. Vasilopoulos, Phys. Rev. B 72, 165336 (2005).

[11] A. Fuhrer, S. Lüescher, T. Ihn, T. Heinzel, K. Ensslin, W. Wegscheider, and M. Bichler, Nature 413, 822 (2001).

[12] A. Tonomura, N. Osakabe, T. Matsuda, T. Kawasaki, J. Endo, S. Yano, and H. Yamada, Phys. Rev. Lett. 56, 792 (1986).

[13] M. König, A. Tschetschetkin, E. M. Hankiewicz, J. Sinova, V. Hock, V. Daumer, M. Schäfer, C. R. Becker, H. Buhmann, and L. W. Molenkamp, Phys. Rev. Lett. 96, 076804 (2006).

[14] A. V. Balatsky and B. L. Altshuler. Phys. Rev. Lett. 70, 001678 (1993).

[15] B. Molnár, F. M. Peeters, and P. Vasilopoulos, Phys. Rev. B 69, 155335 (2004).

[16] S. Souma and B. K. Nikolić, Phys. Rev. Lett. 94, 106602 (2005).

[17] O. Entin-Wohlman, Y. Gefen, Y. Meir, and Y. Oreg, Phys. Rev. B 45, 11890 (1992).

[18] B. H. Wu and J. C. Cao, Phys. Rev. B 74, 115313 (2006).

[19] P. Földi, B. Molnár, M. G. Benedict, and F. M. Peeters, Phys. Rev. B 71, 033309 (2005).

[20] J. Nitta, F. E. Meijer, and H. Takayanagi, Appl. Phys. Lett. 75, 695 (1999).

[21] D. Frustaglia and K. Richter, Phys. Rev. B 69, 235310 (2004).

[22] J. S. Sheng and K. Chang, Phys. Rev. B 74, 235315 (2006).

[23] S. Souma and B. K. Nikolić, Phys. Rev. B 70, 195346 (2004).

[24] B. K. Nikolić and S. Souma, Phys. Rev. B 71, 195328 (2005).

[25] D. S. Fisher and P. A. Lee, Phys. Rev. B 23, 6851 (1981).

[26] S. Datta, Electronic transport in mesoscopic systems (Cambridge University Press, New York, 1997).

[27] L. Yang, M. L. Cohen, and S. G. Louie, Nano. Lett. 7, 3112 (2007).

[28] F. Zhai and H. Q. Xu, Phys. Rev. Lett. 94, 246601 (2005). 